\title{
On Generalized Linear Exponential Distribution: Different Methods of Estimation
}

\author{
M. A. W. Mahmoud ${ }^{1}$, M. G. M. Ghazal ${ }^{2}$, H. M. M. Radwan ${ }^{2 *}$ \\ ${ }^{1}$ Department of Mathematics, Faculty of Science, Al-Azhar University, 11884 Nasr city, Cairo, Egypt \\ ${ }^{2}$ Department of Mathematics, Faculty of Science, Minia University, 61519 Minia, Egypt \\ * Correspondence: hmmradwan86@yahoo.com ; Tel: +201156340041; Fax: +20 862347767
}

\section{Article information}

Received: 29 July 2019

Revised: 12 September 2019

Accepted: 15 September 2019

\section{Key words}

Bathtub hazard rate

Increasing hazard rate

Least square and weighted least square

estimations

Cramér Von-Mises estimation

Anderson Darling estimation

\begin{abstract}
This paper concerns with various techniques for estimations from the generalized linear exponential distribution (GLED) that can be used for modeling bathtub, increasing and decreasing hazard rate (HR) behavior and was first proposed by [3]. This distribution is important since it contains as special sub-models some widely well-known distributions such as the exponential distribution (ED), the Rayleigh distribution (RD), the linear exponential distribution (LED), and the Weibull distribution (WD). The various techniques for estimations can be considered as maximum likelihood estimation (MLE), least-square estimation (LSE), weighted least square estimation (WLSE), Cramér Von-Mises estimation (CVME), and Anderson Darling estimation (ADE). These methods of estimations are used to estimate the unknown parameters of the well-known GLED. Two applications are used to show that the GLED is a viable distribution in modeling lifetime data and to compare the varying methods of estimations based on the Kolmogorov-Simnorov test with the corresponding P-value to show the optimal method. Finally, a simulation study is presented to compare the varying methods of estimation based on the mean square error (MSE) and the average absolute bias (AAB).
\end{abstract}

\section{Introduction}

In studying lifetime data, One can show that the LED, which has only increasing HR, is well-known for modeling lifetime data in reliability analysis. Many authors introduced a generalization of LED to make it a model phenomenon with decreasing, unimodal and bathtub HRF. [1] studied the exponentiated linear exponential distribution (ELED) and showed that the HRF can be increasing, decreasing and bathtub shaped. The transmuted linear exponential distribution with increasing, decreasing, unimodal and bathtub HR shapes were introduced by [2].

Another generalization of LED was known as the generalized LED (GLED) and was proposed first by [3]. Several authors have considered the generalization for the GLED [[4], [5] and [6]]. Recently, [7] provided some notes on GLED in [2] and [8] proposed a new transformation called inverted GLED. The fundamental point of this paper is to study how the various estimators of the unknown parameters of a GLED behave for various sample sizes and different parameter values.

The remainder of the article is organized into six sections. Section 2 introduces some statistical functions of GLED. The MLE of GLED is studied in Section 3. Section 4 studies least square estimator and weighted least square estimator for estimating the parameters of GLED. Analysis of the Cramér Von-Mises estimator using GLED is given in Section 5. Section 6 describes the Anderson Darling estimator. Analysis of two real data sets is presented in Section 7. Section 8 presents a simulation study to compare the different methods of estimation.

\section{Generalized Linear Exponential Distribution}

For a non-negative random variable $X$, the cumulative distribution function (CDF) and the probability density function (PDF) of GLED are given respectively by

$$
F(x ; c, b, \xi)=1-e^{-\left(c x+\frac{b}{2} x^{2}\right)^{\xi}}, \quad c \geq 0, b \geq 0, \xi>0, x>0 .
$$

and

$$
f(x ; c, b, \xi)=\xi e^{-\left(c x+\frac{b}{2} x^{2}\right)^{\xi}}\left(c x+\frac{b}{2} x^{2}\right)^{\xi-1}(c+b x)
$$

The survival and hazard rate functions are respectively by

$$
S(t ; \Phi)=e^{-\left(c t+\frac{b}{2} t^{2}\right)^{\xi}},
$$

and

$$
h(t ; \Phi)=\xi\left(c t+\frac{b}{2} t^{2}\right)^{\xi-1}(c+b t) \quad, \quad t>0,
$$

where $\Phi=(c, b, \xi)$ is the parameter vector.

Remark 1.

For $\xi=1$, Equation (2) reduces to

$$
F(x ; c, b, \xi)=1-e^{-\left(c x+\frac{b}{2} x^{2}\right)}, x>0,
$$

which is the CDF of the LED [9]. If $b=0$ and $c=0$ with $\xi=1$, the ED and the RD are given respectively.

It is observed in [3] that the three-parameter GLED can have increasing, decreasing and bathtub failure rates. 


\section{Maximum Likelihood Estimation}

MLE is likely the most broadly utilized technique for estimation in statistics. Suppose that $x_{1}, \ldots, x_{r}$ be an independent random sample of size $r$ from GLED. From Equation (2), the log-likelihood function can be obtained as

$$
\begin{gathered}
\ell(\Phi)=\mathrm{r} \log (\xi)-\sum_{i=1}^{r}\left(\frac{b}{2} x_{i}^{2}+c x_{i}\right)^{\xi}+(\xi-1) \\
\sum_{i=1}^{r} \log \left(\frac{b}{2} x_{i}^{2}+c x_{i}\right)+\sum_{i=1}^{r} \log \left(b x_{i}+c\right) .
\end{gathered}
$$

By taking the first derivative $\left(\ell_{\Phi}(\Phi)=\frac{\partial \ell}{\partial \Phi}\right)$ of (7) w.r.t. $c, b$, and $\xi$ we get

$$
\begin{aligned}
& \ell_{c}(\Phi)=-\sum_{i=1}^{r} \xi x_{i}\left(\frac{1}{2} b x_{i}^{2}+c x_{i}\right)^{\xi-1}+\sum_{i=1}^{r} \frac{(\xi-1) x_{i}}{\frac{1}{2} b x_{i}^{2}+c x_{i}} \\
&+\sum_{i=1}^{r} \frac{1}{b x_{i}+c}, \\
& \ell_{b}(\Phi)=-\sum_{i=1}^{r} \frac{1}{2} \xi x_{i}^{2}\left(\frac{1}{2} b x_{i}^{2}+c x_{i}\right)^{\xi-1}+\sum_{i=1}^{r} \frac{(\xi-1) x_{i}^{2}}{2\left(\frac{1}{2} b x_{i}^{2}+c x_{i}\right)} \\
&+\sum_{i=1}^{r} \frac{x_{i}}{b x_{i}+c}
\end{aligned}
$$

and

$$
\begin{gathered}
\ell_{\xi}(\Phi)=\frac{\mathrm{r}}{\xi}-\sum_{i=1}^{r}\left(\frac{1}{2} b x_{i}^{2}+c x_{i}\right)^{\xi} \log \left(\frac{1}{2} b x_{i}^{2}+c x_{i}\right) \\
+\sum_{i=1}^{r} \log \left(\frac{1}{2} b x_{i}^{2}+c x_{i}\right),
\end{gathered}
$$

\subsection{The parameters $c$, and $b$ are known}

The normal equation $\ell_{\xi}(\Phi)=0$ can be written as

$\frac{1}{\xi}=\frac{\sum_{i=1}^{r}\left(\frac{b}{2} x_{i}^{2}+c x_{i}\right)^{\xi} \log \left(\frac{b}{2} x_{i}^{2}+c x_{i}\right)-\sum_{i=1}^{r} \log \left(\frac{b}{2} x_{i}^{2}+c x_{i}\right)}{\mathrm{r}}$.

It is clear that the first derivative of the right-side hand $\left(\Psi_{1}(\xi)\right)$ of (11) w.r.t. $\xi$ is always positive. This means that the $\Psi_{1}(\xi)$ is an increasing function. Then by the graphical method [10], the MLE of $\xi$ exists and unique.

\subsection{The parameters $c, b$, and $\xi$ are unknown}

The MLE $\widehat{\Phi}$ of $\Phi$ is given by solving the three normal equations $\ell_{c}(\Phi)=0, \ell_{b}(\Phi)=0$, and $\ell_{\xi}(\Phi)=0$. These nonlinear equations cannot be solved analytically see [1]. So, some numerical technique can be employed to solve these three nonlinear equations.

\subsection{Fisher information matrix}

Since the calculation of Fisher information matrix (given by taking the expectation of the second derivative of (7)) is extremely difficult, so, it appears to be proper to approximate these expected values by their MLEs. Then, the asymptotic variance-covariance matrix is given as [see, [11]];

$$
\begin{aligned}
& \left(\begin{array}{lll}
\operatorname{Var}(\hat{c}) & \operatorname{Cov}(\hat{c}, \hat{b}) & \operatorname{Cov}(\hat{c}, \hat{\xi}) \\
\operatorname{Cov}(\hat{b}, \hat{c}) & \operatorname{Var}(\hat{b}) & \operatorname{Cov}(\hat{b}, \hat{\xi}) \\
\operatorname{Cov}(\hat{\xi}, \hat{c}) & \operatorname{Cov}(\hat{\xi}, \hat{b}) & \operatorname{Var}(\hat{\xi})
\end{array}\right)= \\
& \left(\begin{array}{lll}
-\ell_{c c}(\Phi) & -\ell_{c b}(\Phi) & -\ell_{c \xi}(\Phi) \\
-\ell_{b c}(\Phi) & -\ell_{b b}(\Phi) & -\ell_{b \xi}(\Phi) \\
-\ell_{\xi c}(\Phi) & -\ell_{\xi b}(\Phi) & -\ell_{\xi \xi}(\Phi)
\end{array}\right)_{(\hat{c}, \hat{b}, \hat{\xi})}^{-1}
\end{aligned}
$$

where $\ell_{\Phi_{i} \Phi_{j}}(\Phi)=\frac{\partial^{2} \ell}{\partial \Phi_{i} \Phi_{j}}, i, j=1,2,3$, see Appendix A. Accordingly, the ACIs based on the asymptotic variancecovariance matrix for the parameters $c, b$, and $\xi$ are respectively given as:

$$
\hat{c} \pm Z_{\frac{\alpha}{2}} \sqrt{\operatorname{Var}(\hat{c})}, \hat{b} \pm Z_{\frac{\alpha}{2}} \sqrt{\operatorname{Var}(\hat{b})} \text {, and } \hat{\xi} \pm Z_{\frac{\alpha}{2}} \sqrt{\operatorname{Var}(\hat{\xi})},
$$

where $Z_{\frac{\alpha}{2}}$ is the percentile of the standard normal distribution with right tail probability $\frac{\alpha}{2}$.

\section{Least square and weighted least square estimations}

The study by [12] introduces the LSE and the WLSE for evaluating the estimation of the parameters for beta distribution. These techniques will perform to estimate the parameters of GLED. For this reason, take $x_{i}, i=1, \ldots, r$ as the ordered sample of a random sample of size $r$. At that point, the LSE of the parameters of GLED can be obtained by minimizing the following function

$$
L 1=\sum_{i=1}^{r}\left(F\left(x_{i} ; c, b, \xi\right)-\frac{i}{r+1}\right)^{2}
$$

w.r.t. the unknown parameters $c, b$, and $\xi$ or by solving the following non-linear equations

$$
\begin{aligned}
& \frac{\partial L 1}{\partial c}=\sum_{i=1}^{r} 2\left(1-e^{-\left(c x_{i}+\frac{b}{2} x_{i}^{2}\right)^{\xi}}-\frac{i}{r+1}\right) \delta_{1}\left(x_{i} ; \Phi\right)=0, \\
& \frac{\partial L 1}{\partial b}=\sum_{i=1}^{r} 2\left(1-e^{-\left(c x_{i}+\frac{b}{2} x_{i}^{2}\right)^{\xi}}-\frac{i}{r+1}\right) \delta_{2}\left(x_{i} ; \Phi\right)=0,
\end{aligned}
$$

and

$$
\frac{\partial L 1}{\partial \xi}=\sum_{i=1}^{r} 2\left(1-e^{-\left(c x_{i}+\frac{b}{2} x_{i}^{2}\right)^{\xi}}-\frac{i}{r+1}\right) \delta_{3}\left(x_{i} ; \Phi\right)=0,
$$

where

$$
\begin{gathered}
\delta_{1}\left(x_{i} ; \Phi\right)=\xi \sum_{i=1}^{r} x_{i} e^{-\left(c x_{i}+\frac{b}{2} x_{i}^{2}\right)^{\xi}}\left(c x_{i}+\frac{b}{2} x_{i}^{2}\right)^{\xi-1} \\
\delta_{2}\left(x_{i} ; \Phi\right)=\xi \sum_{i=1}^{r} \frac{1}{2} x_{i}^{2} e^{-\left(c x_{i}+\frac{b}{2} x_{i}^{2}\right)^{\xi}}\left(c x_{i}+\frac{b}{2} x_{i}^{2}\right)^{\xi-1},
\end{gathered}
$$

and

$\delta_{3}\left(x_{i} ; \Phi\right)=\sum_{i=1}^{r} e^{-\left(c x_{i}+\frac{b}{2} x_{i}^{2}\right)^{\xi}}\left(c x_{i}+\frac{b}{2} x_{i}^{2}\right)^{\xi} \log \left[\left(c x_{i}+\frac{b}{2} x_{i}^{2}\right)\right]$,

To determine the WLSE of the unknown parameters of GLED, minimizes the following function

$$
L 2=\sum_{i=1}^{r} \frac{(r+2)(r+1)^{2}}{(r-i+1)}\left(F\left(x_{i}\right)-\frac{i}{r+1}\right)^{2}
$$


w.r.t. the unknown parameters $\Phi$ or solve the non-linear equations

$$
\begin{aligned}
& \frac{\partial L 2}{\partial c}=\sum_{i=1}^{r} 2 \frac{(r+2)(r+1)^{2}}{(r-i+1)}\left(1-e^{-\left(c x_{i}+\frac{b}{2} x_{i}^{2}\right)^{\xi}}-\frac{i}{r+1}\right) \delta_{1}\left(x_{i} ; \Phi\right)=0, \\
& \frac{\partial L 2}{\partial b}=\sum_{i=1}^{r} 2 \frac{(r+2)(r+1)^{2}}{(r-i+1)}\left(1-e^{-\left(c x_{i}+\frac{b}{2} x_{i}^{2}\right)^{\xi}}-\frac{i}{r+1}\right) \delta_{2}\left(x_{i} ; \Phi\right)=0
\end{aligned}
$$

and

$$
\frac{\partial L 2}{\partial \xi}=\sum_{i=1}^{r} 2 \frac{(r+2)(r+1)^{2}}{(r-i+1)}\left(1-e^{-\left(c x_{i}+\frac{b}{2} x_{i}^{2}\right)^{\xi}}-\frac{i}{r+1}\right) \delta_{3}\left(x_{i} ; \Phi\right)=0
$$

where $\delta_{1}\left(x_{i} ; \Phi\right), \delta_{2}\left(x_{i} ; \Phi\right)$ and $\delta_{3}\left(x_{i} ; \Phi\right)$ are given by (12), (13), and (14) respectively.

\section{Cramér Von-Mises estimation}

In a study conducted by [13], it was demonstrated that the bias of CVME is smaller than the other minimum distance estimator. The CVME of GLED can be given by minimizing the following function

$$
W^{2}=\frac{1}{12 r}+\sum_{i=1}^{r}\left(F\left(x_{i}\right)-\frac{2 i-1}{2 r}\right)^{2}
$$

w.r.t. the unknown parameters $\Phi$ or by solving the following non-linear equations

$$
\begin{aligned}
& \frac{\partial W^{2}}{\partial c}=\sum_{i=1}^{r} 2\left(1-e^{-\left(c x_{i}+\frac{b}{2} x_{i}^{2}\right)^{\xi}}-\frac{2 i-1}{2 r}\right) \delta_{1}\left(x_{i} ; \Phi\right)=0, \\
& \frac{\partial W^{2}}{\partial b}=\sum_{i=1}^{r} 2\left(1-e^{-\left(c x_{i}+\frac{b}{2} x_{i}^{2}\right)^{\xi}}-\frac{2 i-1}{2 r}\right) \delta_{2}\left(x_{i} ; \Phi\right)=0,
\end{aligned}
$$

and

$$
\frac{\partial W^{2}}{\partial \xi}=\sum_{i=1}^{r} 2\left(1-e^{-\left(c x_{i}+\frac{b}{2} x_{i}^{2}\right)^{\xi}}-\frac{2 i-1}{2 r}\right) \delta_{3}\left(x_{i} ; \Phi\right)=0,
$$

where $\delta_{1}\left(x_{i} ; \Phi\right), \delta_{2}\left(x_{i} ; \Phi\right)$ and $\delta_{3}\left(x_{i} ; \Phi\right)$ are given by (12), (13), and (14) respectively.

\section{Anderson Darling estimation}

In another study, [14] studied the properties of ADE. Under the results gave by him, the ADE of GLED can be given by minimizing the following function

$$
A^{2}=-r-\sum_{i=1}^{r} \frac{(2 i-1)}{r}\left(\log \left(F\left(x_{i}\right)\right)+\log \left(1-F\left(x_{r-i+1}\right)\right)\right),
$$

w.r.t. the unknown parameters $\Phi$ or by solving the following non-linear equations:

$$
\begin{aligned}
& \frac{\partial A^{2}}{\partial c}=-\sum_{i=1}^{r} \frac{(2 i-1)}{r}\left(\frac{\delta_{1}\left(x_{i} ; \Phi\right)}{1-e^{-\left(c x_{i}+\frac{b}{2} x_{i}^{2}\right)^{\xi}}}-\frac{\delta_{1}\left(x_{r-i+1} ; \Phi\right)}{\left.e^{-\left(c x_{r-i+1}+\frac{b}{2} x_{r-i+1}^{2}\right)}\right)}\right)=0, \\
& \frac{\partial A^{2}}{\partial b}=-\sum_{i=1}^{r} \frac{(2 i-1)}{r}\left(\frac{\delta_{2}\left(x_{i} ; \Phi\right)}{1-e^{-\left(c x_{i}+\frac{b}{2} x_{i}^{2}\right)^{\xi}}}-\frac{\delta_{2}\left(x_{r-i+1} ; \Phi\right)}{e^{-\left(c x_{r-i+1}+\frac{b}{2} x_{r-i+1}^{2}\right)^{\xi}}}\right)=0,
\end{aligned}
$$

and

$$
\frac{\partial A^{2}}{\partial \xi}=-\sum_{i=1}^{r} \frac{(2 i-1)}{r}\left(\frac{\delta_{3}\left(x_{i} ; \Phi\right)}{1-e^{-\left(c x_{i}+\frac{b}{2} x_{i}^{2}\right)^{\xi}}}-\frac{\delta_{3}\left(x_{r-i+1} ; \Phi\right)}{e^{-\left(c x_{r-i+1}+\frac{b}{2} x_{r-i+1}^{2}\right)^{\xi}}}\right)=0,
$$

where $\delta_{1}\left(x_{i} ; \Phi\right), \delta_{2}\left(x_{i} ; \Phi\right)$ and $\delta_{3}\left(x_{i} ; \Phi\right)$ are given by (12), (13), and (14) respectively

\section{7 . Real Data Analysis}

In this section, two real data sets are presented for interpretative study. For identifying the shapes of hazard rate for given data sets, the scaled TTT transform plot is given as

$$
\phi_{r}\left(\frac{n}{r}\right)=\frac{\sum_{i=1}^{r} x_{i: r}+(r-n) x_{n: r}}{\sum_{i=1}^{r} x_{i}},
$$

where $n=1, \ldots, r$ and $x_{i: r}$ is the order statistics of the data [see [15]]. $K-S$ test is used for non-parametric test statistic. This test is defined as:

$$
K-S=\max \left(\left|\frac{i}{r}-F\left(x_{i}\right)\right|,\left|F\left(x_{i}\right)-\frac{i-1}{r}\right|\right) .
$$

For every data set, we compare the different methods of estimation for GLED using the K-S test with the corresponding P-value. All computations were introduced by Wolfram Mathematicall.

\subsection{Lifetimes of 50 devices}

Consider the lifetime data of 50 devices which were provided by [15]. The data set are

$\{0.1,0.2,1,1,1,1,1,2,3,6,7,11,12,18,18,18,18,18,21,32$, $36,40,45,46,47,50,55,60,63,63,67,67,67,67,72,75,79$, $82,82,83,84,84,84,85,85,85,85,85,86,86\}$. All estimates of the unknown parameters based on the different methods, which considered in this paper, and the values of K-S with the value of the corresponding p-value are summarized in (Table 1) for given data. From (Table 1), one can show that the MLE is considered as a good method for given data at a level of significance $\alpha=0.05$. Also, all methods can be considered as good methods for given data at $\alpha=0.01$. It is clear from Table 1 that the MLE is the optimal method under the K-S test with the corresponding $\mathrm{p}$-value. The data are known to have a bathtub-shaped failure rate as shown by the scaled TTTTransform plot, which has a convex shape followed by a concave shape, See Figure (3a). The graphical method of $\xi$ and $\Psi_{1}(\xi)$ based on Equation (11) can be shown in (Figure 1) for given data.

Table 1: The estimates of unknown parameters under varying methods and the $K-S$ test with the corresponding P-value for the lifetime data of 50 devices data

\begin{tabular}{cccccc}
\hline Methods & $\hat{\boldsymbol{c}}$ & $\widehat{\boldsymbol{b}}$ & $\hat{\boldsymbol{\xi}}$ & $\boldsymbol{K}-\boldsymbol{S}$ & $\boldsymbol{P}-$ value \\
\hline MLE & 0.0096 & 0.0004 & 0.7302 & 0.1799 & 0.0786 \\
LSE & 0.0072 & 0.0004 & 0.6457 & 0.2109 & 0.0234 \\
WLSE & 0.0096 & 0.0003 & 1.1484 & 0.1954 & 0.0439 \\
CVME & 0.0075 & 0.0036 & 0.6738 & 0.2040 & 0.0312 \\
ADE & 0.0068 & 0.0004 & 0.5776 & 0.2055 & 0.0293 \\
\hline
\end{tabular}




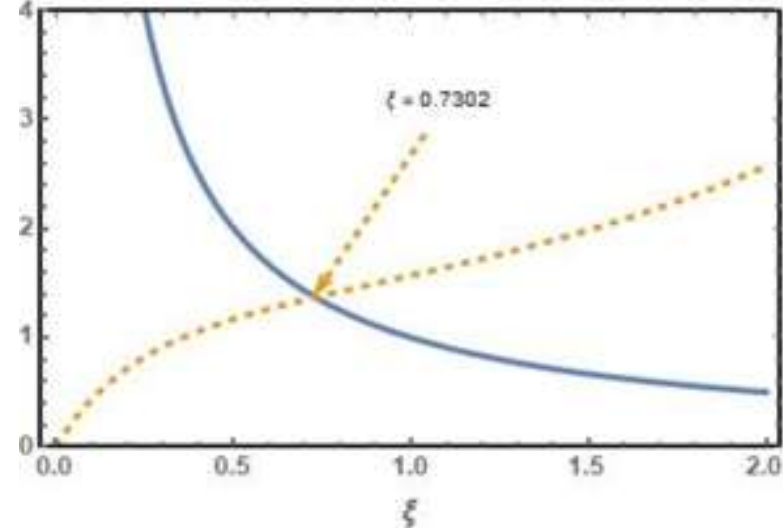

Figure 1: Plot of the $\frac{1}{\xi}$ and $\Psi_{1}(\xi)$ functions for the lifetime data of 50 devices data

\subsection{The electrical appliances failure time data}

Consider the following data set from [16] consisting of the number of cycles to failure for a group of 60 electrical appliances in a life test. The failure times divided by 1000 and detailed as

$\{0.014,0.034,0.059,0.061,0.069,0.08,0.123,0.142,0.165$,

$0.21,0.381,0.464,0.479,0.556,0.574,0.839,0.917,0.969$,

0.991, 1.064, 1.088, 1.091, 1.174, 1.27,1.275, 1.355, 1.397,

$1.477,1.578,1.649,1.702,1.893,1.932,2.001,2.161,2.292$,

2.326,2.337, 2.628, 2.785, 2.811, 2.886, 2.993, 3.122, 3.248,

$3.715,3.79,3.857,3.912,4.1,4.106,4.116,4.315,4.51,4.584$, $5.267,4.299,5.583,6.065,9.701\}$. All estimates of the unknown parameters based on the different methods, which considered in this paper, and the values of K-S with the value of the corresponding p-value are summarized in (Table 2) for given data. From (Table 2), one can show that all methods can be considered as good methods for given data at a level of significance $\alpha=0.05$. It is clear from Table 2 that the ADE is the optimal method under the K-S test with the corresponding pvalue. Furthermore, the TTT plot of the observed data shows that the hazard rate of the electrical appliances failure time data is bathtub which is first convex and then concave as shown in Figure (3b). The graphical method of $\xi$ and $\Psi_{1}(\xi)$ based on Equation (11) can be shown in (Figure 2) for given data.

Table 2: The estimates of unknown parameters under varying methods and the $K-S$ test with the corresponding P-value for the electrical appliances failure time data

\begin{tabular}{cccccc}
\hline Methods & $\hat{\boldsymbol{c}}$ & $\widehat{\boldsymbol{b}}$ & $\hat{\boldsymbol{\xi}}$ & $\boldsymbol{K}-\boldsymbol{S}$ & $\boldsymbol{p}$-value \\
\hline MLE & 0.2185 & 0.1763 & 0.7319 & 0.0632 & 0.9702 \\
LSE & 0.1591 & 0.2029 & 0.6574 & 0.0692 & 0.9361 \\
WLSE & 0.1989 & 0.1789 & 0.7462 & 0.0723 & 0.9123 \\
CVME & 0.1652 & 0.2012 & 0.6787 & 0.0626 & 0.9728 \\
ADE & 0.1692 & 0.2073 & 0.6685 & 0.0604 & 0.9809 \\
\hline
\end{tabular}

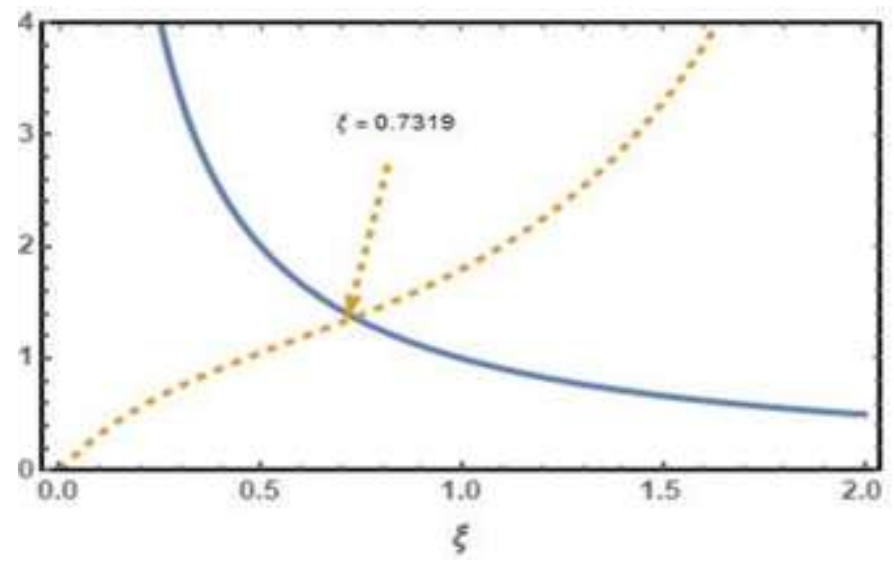

Figure 2: Plot of the $\frac{1}{\xi}$ and $\Psi_{1}(\xi)$ functions for the electrical appliances failure time data.

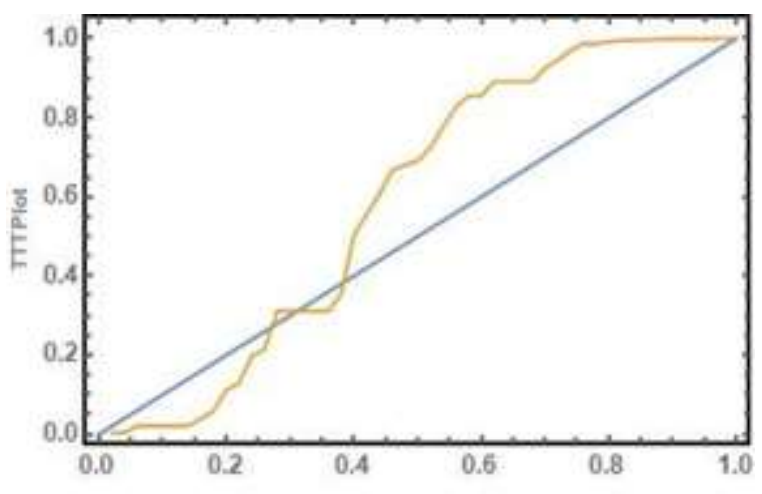

a)

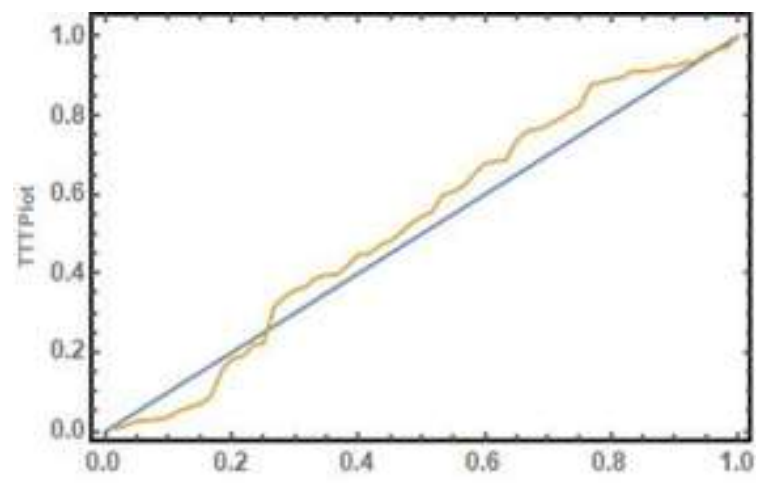

b)

Figure 3: (a) Scaled TTT transform of the Lifetimes of 50 devices data.

(b) Scaled TTT transform of the electrical appliances failure time data.

\section{Simulation Study}

In this section, the simulation study is presented. The MSE and the $\mathrm{AAB}$ are evaluated to study the performance of varying methods of estimation which are given in the previous sections. To compare the performance of varying methods for estimating the unknown parameters of GLED, the following technique is adopted as follows:

1. Set the parameters $(c, b, \xi)$ as $(0.5,0.5,2)$. 
2. Generate random samples from GLED with size $n=$ $(20,50,80,120)$.

3. Evaluate the varying estimates as shown in previous sections.

4. Repeat Steps 1 and $3 N=2000$ times.

5. Calculate the MSEs and AABs.

All numerical values of the simulation study for the different methods of estimation are reported in Tables 3 - 5. From the simulation study, one can show from Tables 3 - 5 that the:

- MSEs for all methods of estimation decrease by increasing the sample size $n$.

- AABs for all methods of estimation decrease by increasing the sample size $n$.

- Based on MSE, all methods of estimation produce smaller MSE for $c$ compared to that of $b$ and $\xi$.

- Based on $\mathrm{AAB}$, all methods of estimation produce smaller $\mathrm{AAB}$ for $c$ compared to that of $b$ and $\xi$.

Table 3: MSEs and AABs for parameter $c$ under proposed methods with different sample size $n$

\begin{tabular}{ccccccc}
\hline $\mathbf{n}$ & & MLE & LSE & WLSE & CVME & ADE \\
\hline $\mathbf{2 0}$ & MSE & 0.1310 & 0.1053 & 0.1089 & 0.1204 & 0.1117 \\
& AAB & 0.3081 & 0.2668 & 0.2721 & 0.2897 & 0.2785 \\
$\mathbf{5 0}$ & MSE & 0.1121 & 0.0935 & 0.1071 & 0.1012 & 0.0941 \\
& AAB & 0.2797 & 0.2441 & 0.2636 & 0.2559 & 0.2438 \\
$\mathbf{8 0}$ & MSE & 0.1066 & 0.0853 & 0.0981 & 0.0921 & 0.0841 \\
& AAB & 0.2657 & 0.2278 & 0.2455 & 0.2377 & 0.2306 \\
$\mathbf{1 2 0}$ & MSE & 0.0933 & 0.0758 & 0.0924 & 0.0816 & 0.0738 \\
& AAB & 0.2410 & 0.2100 & 0.2357 & 0.2183 & 0.2102 \\
\hline
\end{tabular}

Table 4: MSEs and AABs for parameter $b$ under proposed methods with different sample size $n$

\begin{tabular}{ccccccc}
\hline $\mathbf{n}$ & & MLE & LSE & WLSE & CVME & ADE \\
\hline $\mathbf{2 0}$ & MSE & 0.3735 & 0.3536 & 0.3286 & 0.4112 & 0.3715 \\
& AAB & 0.5013 & 0.4570 & 0.4487 & 0.5051 & 0.4824 \\
$\mathbf{5 0}$ & MSE & 0.3139 & 0.2837 & 0.3212 & 0.3101 & 0.2916 \\
& AAB & 0.4492 & 0.4072 & 0.4405 & 0.4311 & 0.4213 \\
$\mathbf{8 0}$ & MSE & 0.3006 & 0.2536 & 0.2884 & 0.2759 & 0.2516 \\
& AAB & 0.4376 & 0.3777 & 0.4093 & 0.3967 & 0.3865 \\
$\mathbf{1 2 0}$ & MSE & 0.2633 & 0.2214 & 0.2668 & 0.2411 & 0.2176 \\
& AAB & 0.3990 & 0.3481 & 0.3913 & 0.3650 & 0.3526 \\
\hline
\end{tabular}

Table 5: MSEs and AABs for parameter $\xi$ under proposed methods with different sample size $\mathrm{n}$

\begin{tabular}{ccccccc}
\hline $\mathbf{n}$ & & MLE & LSE & WLSE & CVME & ADE \\
\hline $\mathbf{2 0}$ & MSE & 0.1670 & 0.2521 & 0.2077 & 0.1901 & 0.2187 \\
& AAB & 0.2952 & 0.3852 & 0.3318 & 0.3095 & 0.3548 \\
$\mathbf{5 0}$ & MSE & 0.1654 & 0.1827 & 0.1963 & 0.1618 & 0.1711 \\
& AAB & 0.3031 & 0.3214 & 0.3325 & 0.2908 & 0.3154 \\
$\mathbf{8 0}$ & MSE & 0.1625 & 0.1517 & 0.1692 & 0.1449 & 0.1453 \\
& AAB & 0.3032 & 0.2887 & 0.3008 & 0.2746 & 0.2871 \\
$\mathbf{1 2 0}$ & MSE & 0.1535 & 0.1369 & 0.1601 & 0.1354 & 0.1337 \\
& AAB & 0.2929 & 0.2721 & 0.2946 & 0.2653 & 0.2737 \\
\hline
\end{tabular}

\section{Conclusion}

In this paper, we compared the estimation of the parameters for the three-parameter distribution IGLED using five estimation methods, namely the maximum likelihood, leastsquares, weighted least-squares, Anderson Darling and Cramér Von-Mises estimations. This conclusion is also supported by the analysis of two real data sets. The K-S test with the corresponding $\mathrm{P}$-value is used to show the optimal method using the two real data. The simulation study is also presented to compare the varying methods using MSEs and AABs.

\section{Acknowledgment}

This paper is a part of the Ph.D. thesis of Hossam Radwan, Department of Mathematics, Faculty of Science, Minia University.

\section{Appendix A.}

The second derivatives of (7) can be written as:

$$
\begin{gathered}
\ell_{c c}(\Phi)=-\sum_{i=1}^{r} \frac{(\xi-1) x_{i}^{2}}{\left(\frac{1}{2} b x_{i}^{2}+c x_{i}\right)^{2}}-\sum_{i=1}^{r} \frac{1}{\left(b x_{i}+c\right)^{2}} \\
-\sum_{i=1}^{r}\left[\begin{array}{lll}
\xi & x_{i}\left(\xi x_{i}\left(\frac{1}{2} b x_{i}^{2}+c x_{i}\right)^{\xi-2}\right. \\
- & \left.\left.x_{i}\left(\frac{1}{2} b x_{i}^{2}+c x_{i}\right)^{\xi-2}\right)\right]
\end{array}\right. \\
\ell_{b b}(\Phi)=-\sum_{i=1}^{r} \frac{(\xi-1) x_{i}^{4}}{4\left(\frac{1}{2} b x_{i}^{2}+c x_{i}\right)^{2}-\sum_{i=1}^{r} \frac{x_{i}^{2}}{\left(b x_{i}+c\right)^{2}}} \\
-\sum_{i=1}^{r}\left[\frac { 1 } { 2 } \xi x _ { i } ^ { 2 } \left(\frac{1}{2} \xi x_{i}^{2}\left(\frac{1}{2} b x_{i}^{2}+c x_{i}\right)^{\xi-2}\right.\right. \\
\left.\left.-\frac{1}{2} x_{i}^{2}\left(\frac{1}{2} b x_{i}^{2}+c x_{i}\right)^{\xi-2}\right)\right] \\
\ell_{\xi \xi}(\Phi)=-\sum_{i=1}^{r}\left(\frac{1}{2} b x_{i}^{2}+c x_{i}\right)^{\xi} \log ^{2}\left(\frac{1}{2} b x_{i}^{2}+c x_{i}\right)-\sum_{i=1}^{r} \frac{1}{(\xi)^{2}}
\end{gathered}
$$




$$
\begin{gathered}
\ell_{c b}(\Phi)=-\sum_{i=1}^{r} \frac{(\xi-1) x_{i}^{3}}{2\left(\frac{1}{2} b x_{i}^{2}+c x_{i}\right)^{2}}+\sum_{i=1}^{r}-\frac{x_{i}}{\left(b x_{i}+c\right)^{2}} \\
-\sum_{i=1}^{r}\left[\xi x _ { i } \left(\frac{1}{2} \xi x_{i}^{2}\left(\frac{1}{2} b x_{i}^{2}+c x_{i}\right)^{\xi-2}\right.\right. \\
\left.\left.-\frac{1}{2} x_{i}^{2}\left(\frac{1}{2} b x_{i}^{2}+c x_{i}\right)^{\xi-2}\right)\right]
\end{gathered}
$$

$$
\begin{aligned}
\ell_{c \xi}(\Phi)=\sum_{i=1}^{r} \frac{x_{i}}{\frac{1}{2} b x_{i}^{2}+c x_{i}}-\sum_{i=1}^{r}\left[x_{i}\left(\frac{1}{2} b x_{i}^{2}+c x_{i}\right)^{\xi-1}\right. \\
\left.+\xi x_{i}\left(\frac{1}{2} b x_{i}^{2}+c x_{i}\right)^{\xi-1} \log \left(\frac{1}{2} b x_{i}^{2}+c x_{i}\right)\right]
\end{aligned}
$$

$$
\begin{aligned}
& \ell_{b \xi}(\Phi)=\sum_{i=1}^{r} \frac{x_{i}^{2}}{2\left(\frac{1}{2} b x_{i}^{2}+c x_{i}\right)}-\sum_{i=1}^{r}\left[\frac{1}{2} x_{i}^{2}\left(\frac{1}{2} b x_{i}^{2}+c x_{i}\right)^{\xi-1}\right. \\
& \left.+\frac{1}{2} \xi x_{i}^{2}\left(\frac{1}{2} b x_{i}^{2}+c x_{i}\right)^{\xi-1} \log \left(\frac{1}{2} b x_{i}^{2}+c x_{i}\right)\right]
\end{aligned}
$$

\section{References}

[1] A. Sarhan and D. Kundu, Generalized linear failure rate distribution, Commun. Stat.-Theor. M., 38, (2009), 642--660.

[2] Y. Tian, M. Tian and Q. Zhu, Transmuted linear exponential distribution: A new generalization of the linear exponential distribution, Commun. Stat. Simul. Comput., 43, (2014), 2661--2677.

[3] M.A.W. Mahmoud and F.M.A. Alam, The generalized linear exponential distribution, Stat. Probab. Lett., 80, (2010), 1005--1014.

[4] A. Sarhan, A.A. Ahmad and I.A. Alasbahi, Exponentiated generalized linear exponential distribution, Appl. Math. Model., 37, (2013), 2838--2849.

[5] H.M. Okasha and M. Kayid, A new family of Marshall-Olkin extended generalized linear exponential distribution, J. Comput. Appl. Math., 296, (2016), 576--592.

[6] M.K. Shakhatreh, A. Yusuf and A-R. Mugdadi, The beta generalized linear exponential distribution, Statistics, 50(6), (2016), 1346--1362.

[7] C.H. Lee and H.J. Tsai, A note on the Generalized linear exponential Distribution, Stat. Probab. Lett., 124, (2017), 49--54.

[8] M.A.W. Mahmoud, M.G.M. Ghazal and H.M.M. Radwan, Inverted generalized linear exponential distribution as a lifetime model, Appl. Math. Inf. Sci., 11(6), (2017), 1747--1765.

[9] L.J. Bain, Analysis for the linear failure-rate life-testing distribution, Technometrics, 16(4), (1974), 551--559.

[10] N. Balakrishnan and M. Kateri, On the maximum likelihood estimation of parameters of Weibull distribution based on complete and censored data, Stat. Probab. Lett., 78, (2008), 2971--2975.

[11] A.C. Cohen, Maximum likelihood estimation in the Weibull distribution based on complete and censored samples, Technometric, 7(4), (1965), 579--588. [12] J. Swain, S. Venkatraman, and J. Wilson, Least squares estimation of distribution function in Johnsons translation system. J Stat Comput Simul., 29, (1988), 271--297.

[13] P. D. M. MacDonald, Comment on "An estimation procedure for mixtures of distributions" by Choi and Bulgren, J. R. Stat. Soc. Series B, Stat. Methodol., 33, (1971), 326--329

[14] D.D. Boos. Minimum distance estimators for location and goodness of fit. J. Am. Stat. Assoc., 76, (1981), 663--670.

[15] M.V. Aarset, How to identify a bathtub hazard rate, IEEE Trans. Rel., 36(1), (1987), 106--108.

[16] J.F. Lawless, Statistical Models and Methods for Lifetime Data, fourth ed. John Wiley, New York. (2003). 